in a general practice survey that the life events, social support and demographic variables which conduced to frank clinical illness also, in lesser doses, made for states of non-specific anxiety and depression. There was a hint of a threshold or 'catastrophe' effect so that a small increase in stressors might lead to a major change in severity of psychopathology. So far, therefore, studies tended to support a unitary concept but conclusions would be premature and a rich research field was available.

Professor E. S. PAYKel pointed out that available evidence indicated that the majority of psychiatric cases within the community saw their general practitioners within the same year, although the disorder might be unrecognized. Studies of general practice consulters could therefore throw light on the qualities of community disorders. He presented data from a study of general practice depression carried out from St. George's with colleagues. Three samples were studied: depressives stanted on a new course of an antidepressant by their GPs; depressives identified by the doctor and given other treatment: and Research Diagnostic Criteria (RDC) major depressives missed by the $\mathrm{GP}$ but identified by screening and subsequent interview. They were studied with multiple rating instruments including the PSE, RDC, Hamilton Depression Scale. The majority were cases on the Index of Definition but predominantly at or just above threshold level and 29 per cent of the other treatment sample were non-cases. Among the antidepressant treated sample, about half were RDC major depsessives and a quarter, minor or intermittent depressives; but among those given other treatment only 20 per cent were major depressives and 30 per cent were minor depressives, while the remainder had anxiety or other diagnoses. The antidepressant treated sample showed considerably less severe depression and less evidence of endogenous symptoms than depressed psychiatric out-patients. The missed major depressives tended to show less overt depressive symptomatology than identified major depressives, but differences were relatively small. Overall the findings indicated that. although it mainly satisfied diagnostic criteria, depression in general practice differed considerably in severity and quality from that treated by psychiatrists.

Ms G. PARRY presented data from the Sheffield Mothers Project (MRC/ESRC Social and Applied Psychology Unit) which studied the effects of paid employment, life event stress and social support on the mental health of working class women with young children. In addition to the PSE as a case identifica. tion procedure, a number of continuously distributed indices of psychological distress were used, including the Beck Depression Inventory. Zung Self-Rated Depression Scale, and measures of somatic anxiety, positive and negative affect and self-esteem. It was found that there was a discontinuity in the linear relationship between the continuously distributed measures and Index of Definition level, between ID4 and ID5. The categorical model was then applied to the data by comparing respondents in ID6 to 8 (definite cases) with those at ID1 to ID4 (non-cases). using discriminant analysis. When items were pre-selected to relate to the psychiatric diagnosis of depression, approximately one-third of ID5 (threshold) respondents were classified as 'cases' using a Bayesian weighted procedure. When using general well-being items, this proportion dropped to 12 per cent and, when using self-attitude items, almost all the threshold cases were within the normal range.

The discussion was opened by Professor D. P. Goldberg. There followed a vigorous discussion involving all speakers and with much audience participation. A particular sense of occasion was imparted by the presence of some of the principal protagonists in a scientific debate which has attracted much attention and it will remain a memorable day for those who attended.

\title{
A New Instrument for Assessment in Rehabilitation Psychiatry
}

REHAB - the Rehabilitation Evaluation Hall and Baker is a recently published method for assessing chronic psychiatric patients. It is intended for use in psychiatric hospitals, day hospitals, hostels and secure institutions. Its authors, John Hall and Roger Baker, are very experienced researchers and practitioners in this ficld and feel that there exists a need in psychiatric rehabilitation for an assessment capable of being applied in a variety of situations. They have therefore constructed REHAB as a multipurpose instrument which can be used: (i) to measure change in patients' behaviour, e.g. for drug trial; (ii) to select in-patients with potential for living in the comunity; (iii) to select disturbed/severely handicapped patients; (iv) to select groups of patients generally: $(v)$ to help plan treatment programmes for individual patients; (vi) to help plan treatment/ intervention/reorganization for a ward, group of wards or whole institution.

REHAB is a package of material containing all that is necessary for assessing up to 50 patients. The entire package consists of a manual for the person administering the assessment, book lets to help raters to understand the assessment, the assessment forms themselves, materials for scoring and recording information and shects designed for presenting the REHAB findings on an individual patient or group of patients. Different items may be replenished as needed.

More detailed information about REHAB, as well as price list and order form, may be obtained from the publishers, Vine Publishing Lid, 2A Eden Place, Aberdeen AB2 4YF, Scotland. A specimen set containing single copies of various parts of the package, retailing at $£ 5.89$ (inclusive of VAT, postage and packing) may also be obtained from Vine Publishing Ltd. 